\title{
Cystectomie totale pour le cancer de la vessie : étude qualitative de l'expérience des patients et implications pour la pratique
}

par Margaret I. Fitch, Debbie Miller, Sharon Sharir et Alison McAndrew

\section{Abrégé}

Les patients subissant des traitements pour le cancer de la vessie font face à des enjeux similaires à ceux des patients atteints d'autres cancers tout en étant confrontés aux enjeux particuliers à leur traitement chirurgical. Cette étude faisait appel à une approche descriptive qualitative en vue d'explorer les expériences des patients ayant subi une cystectomie totale pour leur cancer de la vessie. Vingtdeux patients ont participé à une entrevue en profondeur et ont été invités à assister à une séance de discussion en groupe une fois terminée l'analyse des transcriptions des entrevues individuelles. Les participants décrivaient le choc du diagnostic, le manque d'information sur le cancer de la vessie, l'importance d'une communication claire avec les prestataires de soins ainsi que les diverses adaptations qu'ils avaient dû faire à la suite de la chirurgie. Ainsi, les changements concernant le fonctionnement de l'organisme, l'image corporelle, les relations sexuelles et l'intimité présentaient des défis pour ces participants. Bien qu'un sentiment d'acceptation caractérise les démarches liées au traitement, les patients se devaient de faire des adaptations importantes à la suite de leur chirurgie. L'information, une communication ouverte et le soutien des membres de la famille et des amis étaient autant de facteurs importants au niveau de l'adaptation réussie des patients à la chirurgie. Les patients ont besoin de recevoir une information claire, concise et cohérente sur leur cancer, sur les options de traitement et sur le déroulement des soins. Les infirmières qui soignent des patients ayant subi un traitement chirurgical pour le cancer de la vessie doivent comprendre les besoins particuliers de cette population.

\section{Information générale}

Le cancer de la vessie se classe au sixième rang des tumeurs malignes parmi la population du Canada (Société canadienne du cancer, 2009). Chez les hommes, son incidence est trois fois plus élevée que chez les femmes, mais ces dernières sont de 30 à $50 \%$ plus susceptibles de mourir de la maladie en comparaison avec les hommes (Madeb \& Messing, 2004).

La cystectomie totale est la principale modalité de traitement pour les personnes atteintes d'un cancer de la vessie non invasif sur le plan musculaire et limité à l'organe proprement dit (stade T2) avec ou sans l'ajout d'une chimiothérapie néoadjuvante/adjuvante pour les cancers de la vessie de stade plus avancé (stades T3-4) (Patel \& Campbell). Étant données les différences anatomiques caractérisant le bassin des hommes et des femmes, l'exécution de la chirurgie varie selon le sexe du sujet. Chez les hommes, il y a ablation de la vessie et de la prostate; chez les femmes, il y a, en plus de l'ablation de la vessie et des ganglions lymphatiques pelviens, résection de l'utérus, des ovaires, des trompes de Fallope, de l'urètre et de la paroi antérieure du vagin (Patel \& Campbell, 2009). Pour cette population, les options de reconstruction du tractus urinaire comprennent l'iléostomie (stomie avec dispositif externe), la néovessie (poche interne qui s'évacue naturellement ou est vidangée au moyen d'un cathéter par le biais de l'urètre), ou la poche continente (poche interne s'évacuant par cathéter par stomie au niveau de la paroi abdominale). Du fait de la nature intime de cette procédure, la cystectomie totale peut exercer une incidence significative sur la sexualité (Hart et coll., 1999; Nordström \&
Nyman, 1992; Zippe et coll., 2004). Les difficultés psychosexuelles peuvent influencer à leur tour la qualité de vie globale des patients (Tabano, Condasta \& Coons, 2002; Lagana et coll., 2005).

Un examen de la littérature actuelle révèle un manque relatif d'information sur les expériences de cette population, particulièrement dans le cas des femmes. Les revues systématiques des études comparant la qualité de vie chez les patients ayant subi une cystectomie totale s'accompagnant de divers types de procédures de reconstruction et utilisant un type quelconque d'instrument de mesure entre 1966 et 2008 n'ont pas démontré la supériorité d'un type particulier de dérivation urinaire par rapport aux autres au vu des résultats concernant la qualité de vie (Gerharz et coll., 2005; Porter \& Penson, 2005; Somani, Gimlin, Fayers \& N'dow, 2009). Pour ainsi dire toutes les études examinées utilisaient la méthodologie traditionnelle fondée sur une entrevue clinique, un examen du dossier et un questionnaire dans le but d'évaluer les expériences des patients (Dutta et coll., 2002; Hart et coll., 1999; Henningsohn, Steven, Kallestrup \& Steineck, 2002; McGuire, Grimaldi, Grotas \& Russo, 2000; Nordström \& Nyman, 1992). Une étude utilisait une démarche qualitative afin d'explorer les expériences des patients (Beitz \& Zuzelo, 2003). Toutefois, cette étude s'était bornée à examiner les patients ayant reçu une néovessie et donnait peu de comparaisons entre les expériences des femmes et celles des hommes.

Mais on constate que, même dans les études publiées, fort peu d'attention a été porté à l'unicité potentielle des expériences des femmes subissant ce type de chirurgie. Cela tient peut-être au fait que les hommes sont bien plus nombreux que les femmes à subir ce type de chirurgie, ce qui a donné naissance, chez les urologues, à une culture axée sur le genre masculin (Schover, 2004) ou à la difficulté d'évaluer de manière comparable le dysfonctionnement sexuel chez les hommes et chez les femmes (Nordström \& Nyman, 1992; Porter \& Pendson, 2005). Il se peut que les expériences des femmes et des hommes diffèrent beaucoup pour plusieurs raisons, notamment des différences anatomiques (Porter \& Pendson, 2005) et de possibles différences entre les perspectives masculines et féminines. Il a été suggéré que, par rapport aux hommes, les femmes ont une moindre pulsion sexuelle et une image corporelle plus négative à la suite d'une cystectomie totale (Hart et coll., 1999). De leur côté, Henningsohn et ses collègues (2002) signalent qu'après une cystectomie totale, les femmes présentaient un profil plus similaire à une population témoin appariée que ne le faisaient les

\section{Au sujet des auteures}

Margaret I. Fitch, inf., Ph.D., Centre de cancérologie Odette, 2075 Bayview Ave., Toronto, ON M4N 3M5. Tél. : 416-480-5891; Téléc. : 416-480-7806; Courriel : marg.fitch@sunnybrook.ca

Debbie Miller, inf., B.Sc.inf., M.Sc.inf., ICS(C), Centre de cancérologie Odette, Centre des sciences de la santé Sunnybrook, Toronto, ON.

Sharon Sharir, MD, MHP, FRCSC, Centre de cancérologie Odette, Centre des sciences de la santé Sunnybrook, Toronto, ON.

Alison McAndrew, B.A., RAP, Centre de cancérologie Odette, Centre des sciences de la santé Sunnybrook, Toronto, ON. 
hommes lors d'appariements valables. Une étude qualitative réalisée par Beitz et Zuzelo (2003) rapportait des différences entre les femmes et les hommes concernant leurs expériences. Les femmes exprimaient davantage d'inquiétudes relativement aux cicatrices chirurgicales tandis que les hommes avaient de la difficulté à trouver des endroits propres et appropriés où s'asseoir afin d'évacuer leur urine par voie naturelle ou par cathéter lorsqu'ils se trouvaient dans des toilettes publiques. Comme fort peu de travail a été effectué dans ce domaine, il est impossible de déterminer si les besoins pré- et postopératoires des femmes diffèrent de ceux des hommes dans le cadre du traitement du cancer de la vessie.

\section{But}

Le but de l'étude était de mieux comprendre les expériences des personnes ayant subi une cystectomie totale pour un cancer de la vessie. Nous voulions explorer leurs perspectives relativement à la qualité de vie après la chirurgie en concentrant l'attention sur l'intimité, la sexualité, l'image corporelle et le sens inné de féminité / masculinité. Nous avons demandé aux personnes qui avaient subi cette procédure au cours des 12-36 mois précédents de participer à une entrevue en profondeur. Cette période a été sélectionnée puisqu'elle se caractérise par la stabilisation de la situation postopératoire des patients tout en n'étant pas trop éloignée du moment de l'intervention chirurgicale (Kulaksizoglu, Toktas, Kulaksizoglu, Aglamis \& Unlüer, 2002). Cette étude constituait une exploration libre des expériences des patients en vue de recueillir de l'information sur la sexualité et le concept de soi.

Cette étude est importante parce que fort peu de travaux ont examiné la qualité de vie et la sexualité des patients après une cystectomie totale, que l'on possède bien peu d'information sur les similitudes et les différences entre les deux sexes à ce propos et qu'il n'existait jusqu'alors aucune étude qualitative incluant des patients munis de tous les types de dérivations urinaires. En comprenant mieux les expériences des patients ayant subi une cystectomie totale, nous disposerons de renseignements sur les domaines où des améliorations peuvent être mises en œuvre.

\section{Méthodologie}

\section{Devis}

L'étude faisait appel à une approche descriptive qualitative (Giorgi, 1985; Thorne et coll., 1997) afin d'explorer les expériences des patients ayant subi une cystectomie totale pour un cancer de la vessie. Chaque participant a été interviewé une fois de 12 à 36 mois après sa chirurgie. Ce délai donnait le temps aux patients d'avoir éprouvé les changements associés à la chirurgie et de s'y être adaptés.

\section{Échantillon et procédures}

Avant que l'étude ne soit lancée, son protocole a été approuvé par le comité d'éthique de la recherche de l'hôpital. Quatre-vingt sujets éventuels (40 hommes et 40 femmes) ayant subi une cystectomie totale au cours des 12-36 mois précédents ont été sélectionnés dans la liste de tous les patients atteints d'un cancer de la vessie provenant de la base de données de l'hôpital. Le Bladder Cancer Information System (BLIS) a reçu l'approbation du Comité d'éthique de la recherche de Sunnybrook pour son utilisation à titre de base de données pour la recherche. Avoir au moins 18 ans, bien maîtriser l'anglais et n'avoir aucun antécédent psychiatrique connu constituaient les autres critères d'admissibilité à notre étude.

Le contact initial avec les participants éventuels à l'étude s'est fait par le biais d'une lettre standard signée par leur chirurgien dans laquelle étaient expliqués le but de l'étude, la nature de la participation à l'étude et les prochains contacts envisagés par l'équipe de recherche. L'assistante de recherche a ensuite téléphoné aux patients et leur a fourni davantage de détails sur l'étude, a répondu aux questions des patients et a déterminé si les patients souhaitaient ou non participer à l'étude. Lorsqu'ils acceptaient de le faire, leur consentement écrit a été obtenu au moment de l'entrevue. Le recrutement a été interrompu lorsque nous avons constaté que les entrevues n'engendraient plus aucune nouvelle perspective.

Les données démographiques et cliniques ont été rassemblées en examinant l'information fournie dans la base de données sur le cancer de la vessie tandis que l'information sur les expériences des patients était réunie par le truchement des entrevues. Chaque personne a participé à une entrevue d'environ 60 minutes administrée au moyen d'un guide d'entrevue (voir l'annexe A). L'intervieweuse avait d'excellentes compétences en administration d'entrevues de nature qualitative et une solide formation en recherche psychosociale et psychologique. Les entrevues ont eu lieu dans une salle privée et confortable du centre de cancérologie ou au domicile des patients. La séance entière faisait l'objet d'un enregistrement sonore, et ce dernier a été retranscrit à des fins d'analyse.

Après l'analyse des verbatim des entrevues (dont le résumé est donné ci-dessous), les participants ont été invités à assister à une séance de discussion en groupe durant laquelle les résultats préliminaires de l'analyse ont été présentés. Cette séance permettait aux participants de fournir des renseignements supplémentaires et d'exprimer leurs réactions face aux résultats de l'analyse. Du fait de la nature délicate des sujets explorés, on a tenu un groupe de discussion séparé pour les femmes et un pour les hommes afin que les participants se sentent le plus à l'aise possible.

\section{Guide d'entrevue / guide de groupe de discussion}

Le guide d'entrevue (annexe A) a été élaboré expressément pour cette étude. Ses questions ouvertes visent à faciliter l'exploration des expériences et perceptions des participants concernant : 1) le fait d'être diagnostiqué(e) d'un cancer, 2) le fait de subir une chirurgie pour ce cancer, et 3) l'adaptation aux changements engendrés par la chirurgie. L'intervieweuse se servait du guide afin de veiller à ce que tous les aspects soient abordés. Cependant, les participants pouvaient parler, dès le départ, des sujets qui revêtaient de l'importance à leurs yeux. Des questions incitatives ont été posées seulement si des sujets particuliers (tels que la qualité de vie, la sexualité, l'image corporelle et le sens inné de féminité / masculinité) n'avaient pas été discutés par la personne au cours de la partie initiale de l'entrevue. Même si nous avions prévu que l'entrevue prendrait environ une heure, nous avons donné aux participants tout le temps dont ils avaient besoin pour exprimer ce qu'ils voulaient partager.

Le guide de questions concernant les deux groupes de discussion a lui aussi été élaboré aux fins de la présente étude. Ainsi, les résultats de l'analyse ont été communiqués aux participants au moyen d'une présentation PowerPoint. Puis on a demandé à ces derniers de commenter les résultats relativement à 1) leur pertinence au vu de leur propre expérience, 2) ce qu'ils trouvaient surprenant, le cas échéant, et 3) la clarté de la description des résultats. Les participants ont également été priés d'ajouter tout autre commentaire qu'ils souhaitaient faire à propos des résultats et des recommandations qui devraient être formulées quant aux mesures à prendre. Cette dernière invitation s'articulait particulièrement autour des domaines de l'enseignement aux futurs patients et des conseils à donner aux professionnels de la santé soignant des patients ayant subi une chirurgie pour un cancer de la vessie. Des notes écrites ont été prises durant la séance et celle-ci a également fait l'objet d'un enregistrement sonore.

\section{Analyse des données}

L'analyse des verbatim des entrevues a suivi une démarche d'identification du contenu et des thèmes (Giorgi, 1985; Thorne et coll., Sandelowski, 1997). Les enregistrements sonores ont été retranscrits mot pour mot et les verbatim ont subi une analyse qualitative au moyen du logiciel IN-VIVO. Les membres de l'équipe de recherche ont passé en revue, chacun de leur côté, l'ensemble des 
verbatim, en notant dans la marge les codes s'appliquant éventuellement à la catégorisation du contenu. Ils se sont ensuite réunis afin de discuter de leurs idées sur les codes significatifs en matière de contenu et ont convenu des catégories à utiliser pour organiser ce dernier. Ce schéma de catégorisation a ensuite servi à faire le codage de l'ensemble des verbatim. Les catégories retenues incluaient entre autres " communication », " recherche d'information » et « relations sexuelles ».

Armé de l'information codée pour chaque catégorie de contenu, chacun des membres de l'équipe s'est livré à un examen individuel de ces données et a dégagé les idées principales ou les perspectives communes au sein de chaque catégorie. Puis l'équipe s'est réunie afin de discuter des perspectives cernées et de convenir des notions fondamentales exprimées dans les données. Ces notions ou idées principales devaient rendre les thèmes principaux relatifs aux expériences des patients dont le cancer de la vessie est traité par une intervention chirurgicale.

Cette analyse a ensuite été présentée aux participants des groupes de discussion. Les notes prises lors des réunions des deux groupes de discussion et l'enregistrement sonore ont été examinés par deux des membres de l'équipe. Il leur fallait déterminer si des changements substantiels devaient être apportés à la description préliminaire des résultats et s'il convenait d'y ajouter de nouveaux renseignements.

\section{Rigueur et crédibilité de l'étude proprement dite}

Afin d'assurer la rigueur d'une enquête qualitative, il faut prendre les mesures nécessaires pour que les résultats reflètent adéquatement l'expérience des participants et que les données et leur analyse soient dignes de foi (Connelly \& Yoder, 2000). La rigueur se mesure au moyen des critères suivants : degré de vérité (crédibilité), applicabilité (convenance), cohérence (vérifiabilité), neutralité (impartialité) et enfin, confirmabilité (Lincoln \& Guba, 1985; Sandelowski, 1986; Sandelowski, 1993). Dans la présente étude, la clarification des intentions des chercheuses et le suivi du processus analytique et de la prise de décisions ont donné lieu aux démarches suivantes : (1) les formulaires de consentement et les documents liés à l'étude décrivaient clairement les intentions et les buts de l'étude; (2) les membres de l'équipe de recherche ont couché par écrit les perspectives qu'ils avaient du sujet avant de s'engager dans la collecte ou l'analyse des données; (3) des notes ont été méticuleusement prises tout au long de l'étude en vue de documenter la piste de décisions et les conceptualisations qui ont vu le jour durant la collecte et l'analyse des données; (4) la récapitulation entre collègues (membres de l'équipe de recherche) a permis de discuter de l'élaboration des codes de catégorisation et des thèmes; et enfin, (5) on a cherché des explications concurrentes pour les résultats en vérifiant auprès des participants des deux groupes de discussion et en faisant une recherche dans la littérature. La "valeur de vérité » a été établie (1) en demandant intentionnellement aux participants de parler de leurs perspectives; (2) en demandant aux groupes de discussion de valider les descriptions et l'interprétation des résultats avancées par les chercheuses; et enfin, (3) en présentant les résultats finals aux autres membres de l'équipe de soins aux personnes atteintes de cancer avant d'entreprendre la rédaction du présent article, afin de déterminer le degré d'adéquation entre ces résultats et des contextes autres que celui examiné dans l'étude.

\section{Résultats}

\section{Survol des données démographiques de l'échantillon}

Vingt-deux personnes (treize hommes et neuf femmes) ayant subi une cystectomie totale pour leur cancer de la vessie dans deux établissements ont participé à cette étude (voir le tableau 1). Douze des participants étaient mariés. Douze étaient munis d'une néovessie (NV) et dix avaient une iléostomie (IL). Aucun des participants n'avait de réservoir urinaire continent.

\section{Contenu et thèmes}

Les participants ont donné des descriptions à la fois riches et complètes des expériences qu'ils avaient vécues avant et après leur chirurgie radicale pour un cancer de la vessie. Aux fins du présent article, les résultats globaux seront résumés et enrichis de citations révélatrices des participants. Des descriptions en profondeur de certains aspects des travaux (p. ex. les différences entre les sexes, l'image corporelle, la sexualité) seront élaborées dans le cadre d'articles ultérieurs.

Manque de connaissances sur le cancer de la vessie. Dans l'ensemble, les participants ont exprimé leur manque de compréhension du cancer de la vessie, de ses causes et des facteurs de risque associés et ils jugeaient que cela se traduisait souvent par des retards au niveau de leur diagnostic de cancer. Ils avaient tous éprouvé des symptômes du cancer de la vessie mais n'avaient pas réalisé que ces symptômes pouvaient constituer des signes du cancer. S'ils avaient réalisé la gravité des symptômes, ils auraient probablement consulté un médecin plus tôt qu'ils ne l'avaient fait.

Pour ce qui est des femmes, [les saignements] ne les inquiètent pas plus que ça pour une bonne raison... et mes saignements ont cessé, j'ai donc ignoré la chose et je me suis dit, eh bien...ça ne s'accompagne pas de douleur. (F 74, NV)

Donc je rentrais chez moi en voiture et je me suis dit : "Bon, t'as le cancer, voilà qui intéressant et tu devrais arrêter de fumer. "Je ne sais pas ce que le tabagisme a à voir avec la vessie. (F $75 \mathrm{NV}$ )

Ressentir choc et effroi lors du diagnostic. Tous les participants ont été étonnés d'être diagnostiqués d'un cancer de la vessie. Au début, certains éprouvaient une perte de contrôle sur leur vie tandis que d'autres acceptaient mieux le diagnostic. La plupart ont indiqué avoir le sentiment que la mort leur semblait dorénavant être une réalité plus pressante pour eux.

J'ai été stupéfiée quand j'ai réalisé que c'était de ça qu'il s'agissait. Parce qu'il n'y a pas d'antécédents de cancer dans ma famille ni quoi que ce soit d'autre de la sorte... c'était une surprise totale. (F, 74, NV)

... c'était très pénible, j'avais peur... j'étais très fataliste. Je crois que ce qui doit se passer arrivera. Je veux dire que je n'ai aucun contrôle là-dessus et que je suis entre les mains des médecins et peu importe ce qu'ils veulent que je fasse, je le ferai. (F $64 \mathrm{NV}$ )

Le grand public estime que le cancer représente quasiment une condamnation à mort, tu sais. T'es là et tu penses : je vais mourir. (F $73 \mathrm{IL})$

Tableau 1. Données démographiques des patients

\begin{tabular}{|l|l|l|}
\hline Données démographiques & Hommes $\mathbf{( N = 1 3 )}$ & Femmes (N=9) \\
\hline Patients de Sunnybrook & 11 & 3 \\
\hline $\begin{array}{l}\text { Patients du University } \\
\text { Health Network }\end{array}$ & 2 & 6 \\
\hline Âge moyen & 68,4 & 73,1 \\
\hline Étendue d'âge & $44-82$ & $58-85$ \\
\hline Marié(e)/conjoint(e) de fait & 10 & 2 \\
\hline Veuf/veuve & 0 & 3 \\
\hline Divorcé(e) & 2 & 3 \\
\hline Célibataire & 1 & 1 \\
\hline Iléostomie & 5 & 4 \\
\hline Néovessie & 9 & 4 \\
\hline $\begin{array}{l}\text { Néovessie convertie } \\
\text { en iléostomie }\end{array}$ & 0 & 1 \\
\hline
\end{tabular}


Le besoin d'une communication ouverte. Une communication ouverte avec les professionnels de la santé était perçue comme essentielle par l'ensemble des participants. Beaucoup d'entre eux ont dit que c'était un aspect extrêmement important de leurs interactions avec les prestataires de soins. Ils voulaient que l'information sur le cancer de la vessie et sur les plans de soins soit claire, cohérente d'un professionnel à l'autre et fournie en temps opportun afin d'éviter toute anxiété et confusion. Pour la majorité d'eux, ce n'était pas la manière dont ils avaient reçu de l'information.

Bon, si je comprends bien, il a dit "tumeur » et que c'était une tumeur maligne, mais cela ne signifie-t-il pas "cancer "?...il n'a pas vraiment utilisé le mot "cancer ". Je n'étais pas non plus très satisfaite du traitement que je recevais. Parce que les trois médecins venaient me voir à des moments différents et chacun me disait quelque chose de différent. (F74 NV)

Oh, le niveau de détail, tout ce que je demandais ou tout ce que je voulais savoir [je pouvais le demander]... Je ne parvenais pas à y croire, c'était réellement incroyable, la quantité d'information [mais c'en était accablant]. (H $68 \mathrm{IL})$

Le besoin d'être informés. Le besoin de rechercher des renseignements supplémentaires ou de parler avec des personnes ayant le cancer de la vessie ou portant une dérivation urinaire revêtait de l'importance aux yeux de la plupart des participants, mais c'était une activité qu'ils devaient fréquemment accomplir d'eux-mêmes. Ils pensaient qu'il était utile si des proches et des amis étaient avec eux lorsqu'ils recevaient de l'information des prestataires de soins. Il arrivait souvent que ce soit par le biais de leurs « contacts » avec d'autres personnes ou du "bouche à oreille » qu'ils trouvent des urologues ou des renseignements supplémentaires.

... c'était bien que ma femme soit là [au rendez-vous avec le médecin] parce qu'on pouvait comparer nos notes un peu plus tard... c'est vraiment la seule façon de recevoir de l'information. Je suppose qu'on pourrait aller acheter un gros livre sur le cancer, mais il n'y aurait dedans que deux ou trois paragraphes sur le cancer de la vessie... J'ai découvert un très bon site Web américain... qui s'appelle "Bladder Café » ou un nom dans ce goût-là. (H 44 NV)

Importance du soutien de la famille et des amis. Les participants appréciaient et valorisaient le soutien fourni par la famille et les amis. Beaucoup de patients devaient faire face à de nouvelles expériences et apprendre de nouvelles techniques (élimination et propreté, cathétérisme, changements de dispositifs, etc.).

Je me souviens que j'étais dans la cuisine et je me suis levée, tu sais, [d'un seul coup] le plancher était complètement mouillé [à cause de la fuite]. Il \{son partenaire\} a été celui... il m'a nettoyée. Je suis restée plantée là debout, et les larmes, tu sais... je crois que cela lui faisait du bien de savoir qu'il pouvait faire ça pour moi. (F $74 \mathrm{NV}$ )

Quelques participants sentaient qu'ils n'étaient plus traités de la même façon par les membres de la famille et les amis après le diagnostic et la chirurgie. Ils étaient déçus de cette prise de distance et du manque de soutien qu'ils se croyaient en droit d'attendre.

Les amis vont et viennent et certains d'entre eux ont dépassé nos attentes mais d'autres en étaient bien loin. (H $44 \mathrm{NV}$ )

Période de rétablissement initiale. De manière générale, les soins hospitaliers post-chirurgie étaient perçus comme une bonne expérience. Les participants étaient étonnés de ne pas ressentir davantage de douleur et ont trouvé qu'ils arrivaient à bien se débrouiller. Par contre, l'expérience des soins à domicile était bien plus variable. Des facteurs tels que le lieu de résidence de la personne (c.-à-d. la distance entre lui et l'hôpital), les connaissances du personnel infirmier de leur district au sujet de la procédure chirurgicale et des soins nécessaires (p. ex. pansements, cathétérisme) et l'aide disponible de proches et d'amis influençaient l'expérience des soins à domicile. Composer avec les situations imprévues et les réactions émotionnelles était source de détresse pour certains participants.

Alors, tu comprends, t'es un peu mal à l'aise... T'es couchée là sur le lit et tu réalises que ta vessie est partie pour de bon, tu sais. Je commençais à ne plus avoir le moral. Mais les infirmières m'ont réconfortée... Elles étaient toutes très bien. (F $83 \mathrm{IL}$ )

Les soins à domicile, pas une seule personne ne savait, n'avait une quelconque expérience de ceci. Ils avaient de l'expérience en matière d'intestins, mais pas de la vessie. (F 73 IL)

Composer avec l'incontinence. L'incontinence urinaire ou les fuites d'un dispositif à poche constituaient un problème et une frustration de taille pour beaucoup. Les sujets essayaient plusieurs stratégies ou manières différentes de composer avec la situation. Toutefois, ces essais étaient fréquemment faits de leur propre chef car ils n'avaient reçu aucun enseignement sur ce qu'il convenait de faire en cas d'incontinence ou de fuite. De plus, les professionnels de la santé œuvrant dans la collectivité possédaient rarement les connaissances exigées par ce défi postopératoire.

Tu sais, tu ne veux pas passer le reste de ta vie à mouiller ton pantalon...mais tu ne veux pas mourir non plus. Il faut donc que tu manifestes une certaine ouverture [d'esprit]. (H $44 \mathrm{NV}$ )

Je ne suis pas heureuse de devoir déambuler toute la journée avec une serviette. Je veux dire, je croyais qu'il y avait longtemps que j'en avais fini avec cette imposition-là. (F 64 NV)

Je ne pouvais rien faire [concernant les fuites], tu sais, j'ai donc acheté tous mes pantalons de couleur noire afin que les fuites ne se remarquent pas jusqu'à ce que je sois de retour à la maison. Tout cela est bien gênant et une vraie source de complications. (F 73 IL)

S'adapter aux changements en matière d'image corporelle et de fonctionnement de l'organisme. Tous les participants ont éprouvé, après la chirurgie, des changements sur le plan de l'image corporelle et $\mathrm{du}$ fonctionnement de l'organisme. Ceux-ci étaient plus prononcés chez les participants ayant subi une iléostomie ou étant plus jeunes. La bonne adaptation aux changements semblait associée à l'âge, à l'expérience de vie et au degré d'aise vis-à-vis du corps avant la chirurgie. La plupart des participants ont exprimé un sentiment d'acceptation de la réalité de leur situation et des changements connexes. En revanche, ils ont tous indiqué qu'il leur a fallu fournir de gros efforts pour s'ajuster aux changements et s'accommoder de leur nouvelle vie du mieux qu'ils pouvaient.

Jamais, au grand jamais je ne laisserais quiconque près de mon corps, avec cette stomie et cette poche et tout le bazar - et puis mon estomac, oh, comme je suis dégoûtante. Ça n'a pas d'allure... je veux dire, j'ai une poche de pipi accrochée au ventre. Je trouve ça dégueulasse, tu sais. Je suis sûre que tout le monde ressentirait la même chose! (F 73 IL)

T'as quelque chose qui t'es collé au corps pour toujours et tu sais, il n'y a vraiment pas moyen [de ne pas le porter]. J'essaie depuis longtemps de trouver un moyen de l'enlever pendant une journée mais il semblerait que ce soit impossible. (H 79 IL)

Modifications des relations sexuelles. Le sentiment d'acceptation des modifications sur le plan des relations et du fonctionnement sexuels était influencé par l'âge de la personne et le stade de vie dans lequel elle se voit, par le type de chirurgie et les changements résultants sur le plan du fonctionnement corporel et quotidien, par l'importance que le couple accordait aux relations sexuelles physiques auparavant et si la personne était ou non dans une relation à long terme. Quoiqu'il y avait des défis, les participants ont décrit la valeur de l'intimité au sein du couple et comment cette dernière s'était maintenue ou s'était même améliorée au long de l'expérience du cancer. 
Parce que je ne veux pas... c'est [les rapports sexuels] très inconfortable avec ça. Je ne peux pas m'empêcher de tousser et donc, tu sais [d'avoir des fuites]. Le moins que je puisse dire, c'est que je ne les trouve guère alléchants. (F $64 \mathrm{NV}$ )

... Si c'est ton mari et qu'il t'aimait auparavant... il t'aimera encore. Sinon, je ne crois pas qu'un homme voudra sortir avec une femme qui a un trou dans l'estomac et, tu sais, qui porte une poche d'urine sur la jambe. (F 58 IL)

Je ne me suis jamais senti dépendant, mais alors pas du tout. Je crois que ma femme accepte le fait que cela fasse partie de la vie. Que du point de vue sexuel, ce n'est pas la chose la plus importante... Je ne pense pas que [la chirurgie] ait eu un quelconque impact... je crois même qu'elle a amélioré l'intimité, tu sais. Nous pouvons en parler plus librement, plus ouvertement. (H 64 NV)

Altération des perspectives d'avenir. La vie de tous les participants a été chamboulée du fait de leur diagnostic de cancer et de la chirurgie afférente. Beaucoup ont dit avoir le sentiment que leur vie ne serait plus jamais pareille et qu'il leur fallait composer avec son évolution et la garder la plus normale possible. Ils étaient nombreux à réaliser que leurs priorités avaient changé de même que leurs perspectives sur ce qui compte dans la vie.

Tu réalises que tu ne seras pas là pour l'éternité. Tu réalises que t'es entré dans le dernier quart de ta vie. Tu comprends ce qui compte véritablement. Tu réalises l'importance que revêtent ta famille, tes enfants. Je veux dire que j'ai toujours ressenti ça, mais maintenant c'est passé au tout premier plan. (H 60 NV)

\section{Groupe de discussion}

Six personnes (5 hommes, 1 femme) ont participé aux deux séances de discussion en groupe tenues à titre de suivi (voir le tableau 2). Les participants ont indiqué que les résultats qui leur ont été présentés reflétaient leurs expériences et que celles-ci avaient fait l'objet d'une caractérisation appropriée par l'équipe de recherche. Lors d'une discussion ultérieure, les participants ont souligné que le grand public avait besoin de davantage d'information sur le cancer de la vessie. De leur avis, trop peu d'attention était accordée à l'information du public quant aux signes et symptômes du cancer de la vessie ou aux facteurs de risque connexes. En ce qui concerne les patients, il fallait fournir davantage d'information sur ce à quoi ils doivent s'attendre en phase postopératoire. Ils souhaitaient obtenir de l'information, entre autres, sur la progression du régime alimentaire, le soin des cicatrices, les infections, les soins à domicile, les plans de soins de suivi et la surveillance du cancer. Les participants estimaient qu'il devrait y avoir divers moyens de distribuer cette information notamment les suivants : affiches, livrets, campagnes de sensibilisation, trousses pédagogiques destinées aux patients.

Finalement, les conseils qu'ils adressaient aux professionnels de la santé étaient axés sur l'obligation qu'avaient ces derniers de mieux comprendre les expériences que vivent les patients. Selon eux, les patients bénéficieraient d'une aide élargie si les professionnels de la santé avaient une connaissance plus étendue des diverses options chirurgicales et de l'impact sur la personne des changements corporels afférents. En outre, les participants déclaraient qu'il est essentiel qu'il existe une communication ouverte entre eux et les prestataires de soins, notamment que des réponses soient apportées à leurs questions et que soient explorés leurs problèmes et inquiétudes concernant leur image corporelle individuelle et les changements en matière de sexualité.

\section{Discussion et implications pour la pratique}

Cette étude a été entreprise afin de décrire les expériences des personnes ayant subi une cystectomie totale pour leur cancer de la vessie. Les résultats éclairent la nature des enjeux auxquels sont confrontées les personnes subissant une telle opération radicale et cherchant à s'adapter aux changements sur le plan de l'image et du fonctionnement corporels. Elles partagent certains de ces enjeux avec les patients atteints d'autres cancers tandis que d'autres concernent uniquement ce type de cancer et ces interventions chirurgicales. Cette étude reflète uniquement les travaux préliminaires réalisés auprès de cette population particulière et souligne clairement la nécessité de mener des recherches additionnelles.

Ces travaux révèlent essentiellement qu'il convient de fournir aux patients une meilleure assistance sur le plan de l'information et de l'accès aux ressources. Il est nécessaire d'améliorer et d'élargir le matériel pédagogique sur le cancer de la vessie à l'intention des patients, sur les options relatives à la reconstruction et sur le rétablissement post-chirurgical. De plus, les prestataires de soins doivent être informés de l'importance que revêt la communication ouverte en ce qui concerne : l'expérience du cancer des patients; les options chirurgicales offertes lors de la réalisation d'une cystectomie totale; les adaptations aux atteintes à l'image corporelle, au fonctionnement de l'organisme, à l'intimité et à la sexualité.

Les résultats de notre étude nous amènent à vouloir élaborer un outil d'éducation des patients expliquant les différentes options possibles en matière de reconstruction en cas de cystectomie totale. Nous espérons ainsi rehausser les connaissances globales des patients relativement aux options chirurgicales offertes et faciliter la prise de décision quant à l'intervention chirurgicale qui répond le mieux à leurs besoins et style de vie individuels. Nous avons l'intention de fournir un enseignement au personnel infirmier sur les défis que doivent relever les patients et sur la façon dont il est possible de fournir un soutien accru aux patients et à leurs proches. Nous produirons également du matériel qui facilitera l'enseignement aux patients concernant la gestion de problèmes tels que les fuites et l'incontinence. Pour terminer, le partage de cette information et des plans de soins postopératoires avec les infirmières en soins communautaires rehaussera la continuité des soins lorsque les patients passent des soins en établissement aux soins à domicile et fournira de précieux renseignements aux infirmières soignant cette population aux besoins complexes.

\section{Tableau 2. Groupes de discussion}

\begin{tabular}{|l|l|l|}
\hline Données démographiques & Hommes $(\mathrm{N}=5)$ & Femmes $(\mathrm{N}=1)$ \\
\hline Patients de Sunnybrook & 4 & 0 \\
\hline $\begin{array}{l}\text { Patients du University } \\
\text { Health Network }\end{array}$ & 1 & 1 \\
\hline Âge moyen & 72 & 64 \\
\hline Étendue d'âge & $60-82$ & n. a. \\
\hline Marié(e)/conjoint(e) de fait & 4 & 1 \\
\hline Veuf/veuve & 0 & 0 \\
\hline Divorcé(e) & 1 & 0 \\
\hline Célibataire & 0 & 0 \\
\hline $\begin{array}{l}\text { Iléostomie } \\
\text { Néovessie }\end{array}$ & 1 & 0 \\
\hline $\begin{array}{l}\text { Avaient consenti à participer : } 13 \\
\text { Est revenu sur sa décision de participer : } 1 \text { (femme munie } \\
\text { d'une néovessie) } \\
\text { Ne s'est pas présenté : } 1 \text { (homme porteur d'une iléostomie) }\end{array}$ \\
\hline
\end{tabular}


Annexe A. Guide d'entrevue

Durant l'entretien que nous allons avoir aujourd'hui, j'aimerais que vous me parliez de plusieurs aspects de votre expérience du cancer, à savoir :

1. le fait d'être diagnostiqué(e) du cancer

2. l'opération chirurgicale

3. ce qui s'est passé depuis cette intervention, particulièrement les changements qui résultent de l'opération chirurgicale exigée par votre cancer

1. Pour commencer, j'aimerais savoir ce qui vous a amené(e) à être diagnostiqué(e) du cancer.

QUESTIONS INCITATIVES : Qu'avez-vous remarqué en premier qui indiquait que quelque chose n'allait pas?

Qui a diagnostiqué votre cancer? Comment celui-ci a-t-il été diagnostiqué? Quand a-t-il été diagnostiqué?

2. Quelles actions / activités ont eu lieu en ce qui concerne la chirurgie?
QUESTIONS INCITATIVES : Qui vous a

dit qu'elle était nécessaire?

Aviez-vous suffisamment d'information à son sujet?

Qui participait au processus de prise de décisions?

Quand la chirurgie a-t-elle eu lieu?

Comment les choses se sont-elles

passées à l'hôpital?

Qu'est-ce qu'on vous a fait durant

l'intervention chirurgicale?

Comment les choses se sont-elles passées chez vous juste après l'intervention?

3. Quels changements avez-vous remarqués depuis lors qui, selon vous, résultent de la chirurgie? QUESTIONS INCITATIVES : Qu'est-ce qui n'est plus pareil qu'avant la chirurgie? Que pouvez-vous faire/que ne pouvezvous pas faire à cause de votre chirurgie?

Que ressentez-vous vis-à-vis de ces changements?
Quel impact ces changements

ont-ils eu :

sur vous?

sur ce que vous ressentez à propos

de vous-même?

sur ce que vous ressentez à propos

de votre corps?

sur votre sens de l'identité?

sur votre sentiment de féminité /

masculinité?

4. Étant donné le type de chirurgie que vous avez subi, des changements peuvent survenir sur le plan de l'intimité et/ou des relations sexuelles. Quelle est votre expérience dans ce domaine depuis l'intervention chirurgicale?

5. Y a-t-il quoi que ce soit d'autre dont vous aimeriez me parler au sujet de vos expériences du cancer et de votre vie depuis le diagnostic et la chirurgie?

\section{MERCI DE VOTRE PARTICIPATION!}

\section{Références}

Beitz, J.M., \& Zuzelo, P.R. (2003). The lived experience of having a neobladder. Western Journal of Nursing Research, 25(3), 294-316.

Connelly, L.M., \& Yoder, L.H. (2000). Improving qualitative proposals: Common problem areas. Clinical Nurse Specialist, 1(2), 69-74.

Dutta, S.C., Chang, S.C., Coffey, C.S., Smith, J.A. Jr., Jack, G., \& Cookson, M.S. (2002). Health related quality of life assessment after radical cystectomy: Comparison of ileal conduit with continent orthotopic neobladder. Journal of Urology, 168(1), 164-167.

Gerharz, E.W., Mansson, A., Hunt, S., Skinner, E.C., \& Mansson, W. (2005). Quality of life after cystectomy and urinary diversion: An evidence-based analysis. Journal of Urology, 174(5), 1729-1736.

Giorgi, A. (1985). Sketch of a phenomenological method. In A. Giorgi (Ed.), Phenomenology and Psychological Research (pp. 8-22). Pittsburgh, PA: Pittsburgh Duquesne University Press.

Hart, S., Skinner, E.C., Meyerowitz, B.E., Boyde, S., Lieskovsky, G., \& Skinner, D.G. (1999). Quality of life after radical cystectomy for bladder cancer in patients with an ileal conduit, cutaneous or urethral Kock pouch. Journal of Urology, 162(1), 77-81.

Henningsohn, L., Steven, K., Kallestrup, E.B., \& Steineck, G. (2002). Distressful symptoms and well-being after radical cystectomy and orthotopic bladder substitution compared with a matched control population. Journal of Urology, 168(1), 168-174.

Kulaksizoglu, H., Toktas, G., Kulaksizoglu, I.B., Aglamis, E., \& Unlüer, E. (2002). When should quality of life be measured after radical cystectomy? European Urology, 42(4), 350-355.

Lagana, L., Classen, C., Caldwell, R., McGarvey, E.L., Baum, L.D., Cheasty, E., et al. (2005). Sexual difficulties of patients with gynecological cancers. Professional Psychology: Research \& Practice, 36(4), 391-399.

Lincoln, Y.S., \& Guba, E. (1985). Naturalistic Inquiry. Thousand Oaks, CA: Sage Publications Inc.

Madeb, R., \& Messing, E.M. (2004). Gender, racial and age differences in bladder cancer incidence and mortality. Urologic Oncology, 22(2), 86-92.

McGuire, M.S., Grimaldi, G., Grotas, J., \& Russo, P. (2000). The type of urinary diversion after radical cystectomy significantly impacts on the patient's quality of life [see comment]. Annals of Surgical Oncology, 7(1), 4-8.
Nordström, G.M., \& Nyman, C.R. (1992). Male and female sexual function and activity following ileal conduit urinary diversion. British Journal of Urology, 70(1), 33-39.

Patel, A.R., \& Campbell, S.C. (2009). Current trends in the management of bladder cancer. Journal of Wound, Ostomy and Continence Nursing, 36(4), 413-421.

Porter, M.P., \& Penson, D.F. (2005). Health-related quality of life after radical cystectomy and urinary diversion for bladder cancer: A systematic review and critical analysis of the literature. Journal of Urology, 173, 1318-1322.

Sandelowski, M. (1986). The problem of rigor in qualitative research. Advances in Nursing Science, 8(3), 27-37.

Sandelowski, M. (1993). Rigor or rigor mortis: The problem of rigor in qualitative research revisited. Advances in Nursing Science, 16(2), 1-8.

Sandelowski, M. (1995). Sample size in qualitative research. Research in Nursing \& Health, 18(2) 179-183.

Sandelowski, M. (1997). "To be of use": Enhancing the utility of qualitative research. Nursing Outlook, 45(3), 125-132.

Schover, L.R. (2004). Sexuality after treatment of pelvic cancer (pp. 186-195). Linthicum, MD: American Urological Association.

Société canadienne du cancer / Institut national du cancer du Canada (2009). Statistiques canadiennes sur le cancer 2009. Toronto, ON : Auteur.

Somani, B.K, Gimlin, D., Fayers, P., \& N'dow, J. (2009). Quality of life and body image for bladder cancer patients undergoing radical cystectomy and urinary diversion-A prospective cohort study with a systematic review of literature. Urology, 74(5), 1138-43.

Tabano, M., Condosta, D., \& Coons, M. (2002). Symptoms affecting the quality of life in women with gynecologic cancer. Seminars in Oncology Nursing, 18(3), 223-230.

Thorne, S., Kirkham, S.R., \& MacDonald-Emes, J. (1997). Interpretive description: A noncategorical qualitative alternative for developing nursing knowledge. Research in Nursing \& Health, 20(2), 169-177.

Zippe, C.D., Raina, R., Shah, A.D., Massavayi, E.Z., Agarwal, A., Ulchaker, J., et al. (2004). Female sexual dysfunction after radical cystectomy: A new outcome measure. Urology, 63(6), 1153-1157. 